\title{
Results from proton-lead and fixed-target collisions at $\mathrm{LHCb}$
}

\section{Óscar Boente García*, on behalf of LHCb Collaboration}

Instituto Galego de Física de Altas Enerxías (IGFAE), Universidade de Santiago de Compostela

E-mail: oscar.boentedusc.es

The latest results from the $\mathrm{LHCb}$ experiment in heavy-ion collisions are reported. A variety of different collision systems is covered: proton-lead $(p-\mathrm{Pb})$ collisions at $\sqrt{s_{\mathrm{NN}}}=5.02,8 \mathrm{TeV}$; leadlead $(\mathrm{Pb}-\mathrm{Pb})$ collisions at $\sqrt{s_{\mathrm{NN}}}=5.02 \mathrm{TeV}$ and proton-gas collisions in fixed-target configuration. These measurements exploit the forward geometry of the detector (pseudo-rapidity between 2 and 5) and thus are highly complementary to other measurements at the LHC. The measurements in $p$ - $\mathrm{Pb}$ include the study of different heavy-flavour states, notably $B$ hadrons, $\Upsilon(n S), \Lambda_{c}^{+}$ and coherent $J / \psi$ production in ultra-peripheral $\mathrm{Pb}-\mathrm{Pb}$ collisions. In fixed-target configuration, $\bar{p}$ production in $p$-He collisions and charm production in $p$-He and $p$-Ar are covered.

XXVII International Workshop on Deep-Inelastic Scattering and Related Subjects - DIS2019 8-12 April, 2019

Torino, Italy

${ }^{*}$ Speaker. 


\section{Introduction}

LHCb is one of the four main experiments located at the Large Hadron Collider at CERN [1]. The detector is a forward spectrometer fully instrumented between 2 and 5 units of pseudo-rapidity $(\eta)$, where the production of $\mathrm{b}$ and $\mathrm{c}$ hadrons is maximal. It is instrumented with a vertex locator (VELO), a tracking system with excellent transverse momentum $\left(p_{\mathrm{T}}\right)$ resolution, two ring-imaging Cherenkov detectors (RICH) that provide excellent charged hadron identification, calorimetry and a muon system [2].

The peculiarities of the detector grant the possibility to make a key contribution to the understanding of heavy-ion physics. Firstly, the relatively uncovered detector acceptance, fully in the forward region, is highly complementary to other LHC experiments. Secondly, the detector has an outstanding reconstruction performance for exclusive heavy flavour states, being able to reach null transverse momentum with an excellent mass resolution. Thirdly, LHCb has the capability to disentangle the contribution of prompt particles and those originated in decays from B hadrons, thanks to the identification of secondary vertexes with the VELO.

The detector is able to fully exploit these capabilities in $p$ - $\mathrm{Pb}$ collisions. More challenging is the study of $\mathrm{Pb}-\mathrm{Pb}$ collisions due to the high multiplicities reached, where saturation of the tracking system is observed. Therefore LHCb cannot operate currently in the most central Pb$\mathrm{Pb}$ collisions. However, the performances of the experiment allow to study ultra-peripheral and peripheral collisions, which are those where the nuclei do not overlap completely.

In addition, $\mathrm{LHCb}$ can operate as a fixed-target experiment, thanks to its fixed-target like geometry. This is achieved with the injection of low pressure noble gas in the LHC vacuum [3]. The resulting beam-gas collisions are then studied, covering a new unexplored regime in centre-ofmass energies.

\section{Collider configuration: proton-lead and lead-lead collisions}

The study of proton-lead collisions is the cornerstone of the heavy-ion physics program at LHCb. The reach in pseudo-rapidity is further extended by reversing the direction of the proton and lead beams, accessing the forward $(\eta>0)$ and the backward $(\eta<0)$ pseudo-rapidity regions in the centre-of-mass system. Depending on the probed particles, one can access the nuclear parton distribution functions (nPDFs) at low Bjorken- $x$ and medium Bjorken- $x$ in the forward and backward configurations, respectively. Data from $p-\mathrm{Pb}(\mathrm{Pb}-p)$ have been acquired in two data-taking periods, a first run in 2013 at $\sqrt{s_{\mathrm{NN}}}=5.02 \mathrm{TeV}$ with $1.1 \mathrm{nb}^{-1}\left(0.4 \mathrm{nb}^{-1}\right)$ of integrated luminosity in the forward (backward), and a second run in 2016 at $\sqrt{s_{\mathrm{NN}}}=8.16 \mathrm{TeV}$ with $12.5 \mathrm{nb}^{-1}\left(17.4 \mathrm{nb}^{-1}\right)$.

With these datasets, many measurements of the production of heavy-flavour states have been performed. For instance, one can measure the nuclear modification factor $R_{p \mathrm{~Pb}}$, defined as the ratio of the cross-sections in $p-\mathrm{Pb}$ and $p-p$ normalised to the nucleon number. This factor is sensitive to Cold Nuclear Matter (CNM) effects, which include a large number of phenomena such as energy loss [4], nPDFs [5] or interactions with the co-moving medium [6].

LHCb has measured for the first time the exclusive production of $b$-hadrons in nuclear collisions [7]. Several decay modes are used, in particular $B^{+} \rightarrow \bar{D}^{0} \pi^{+}, B^{+} \rightarrow J / \psi K^{+}, B^{0} \rightarrow D^{-} \pi^{+}$ and $\Lambda_{b}^{0} \rightarrow \Lambda_{c}^{+} \pi^{-}$, to reconstruct three different states and study double differential cross-sections, 

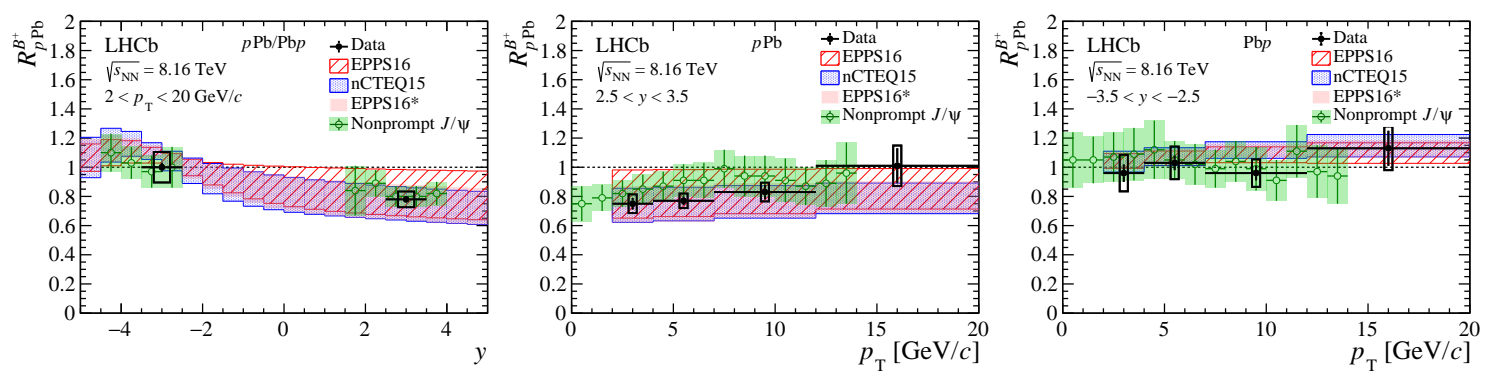

Figure 1: Nuclear modification factors for the $B^{+}$meson [7]. The measurement is presented as a function of pseudo-rapidity (left plot) and as a function of transverse momentum in the forward region (center) and backward region (right plot). The $R_{p \mathrm{~Pb}}$ (black) is compared with predictions from different sets of nPDFs and with the measurement of the detached $J / \psi$ from $b$ decays [8]
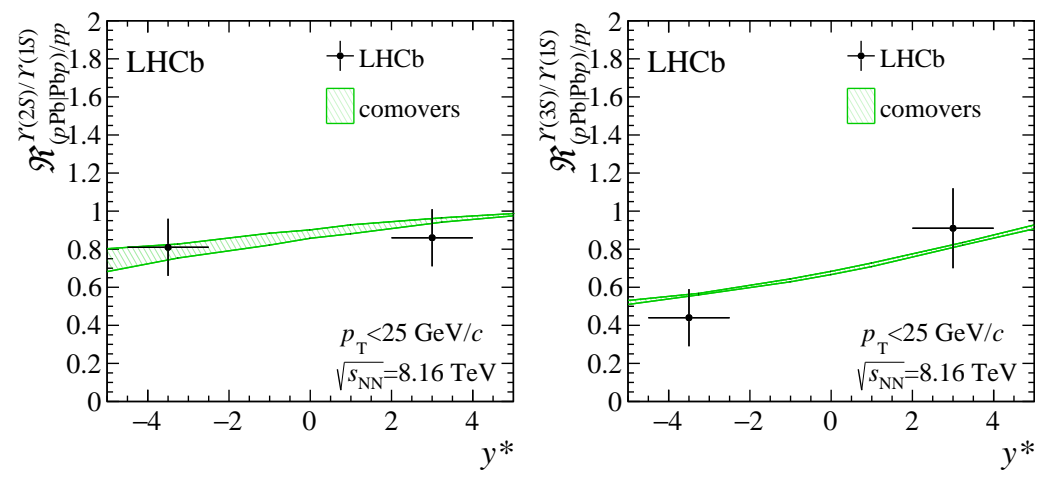

Figure 2: Ratio between the $\Upsilon$ nuclear modification factors as a function of rapidity for $\Upsilon(2 S) / \Upsilon(1 S)$ (left plot) and $\Upsilon(3 S) / \Upsilon(1 S)$ (right plot) [9].

nuclear modification factors, and forward-to-backward ratios. In figure 1, the nuclear modification factors for the $B^{+}$are shown. The measured values agree with the predictions from nPDFs and with the previously measured non prompt $J / \psi$ modification [8], confirming the observed suppression pattern.

Based also on the $2016 p$ - $\mathrm{Pb}$ data-taking, the production of the different $\Upsilon(n S)$ states has been studied [9]. In the LHCb measurement the states $\Upsilon(1 S), \Upsilon(2 S)$ and $\Upsilon(3 S)$ are cleanly observed and the nuclear modification can be measured. Figure 2 shows the ratio between the nuclear modification factors of the different upsilon states. The observed relative suppression is possibly due to the interaction between final-state particles as described by the "comovers" model [6].

Additionally, the $\Lambda_{c}^{+}$baryon production can be studied already with lower integrated luminosity using the 2013 sample [10]. The state is reconstructed with $\Lambda_{c}^{+} \rightarrow p K^{-} \pi^{+}$decay to measure the forward-to-backward ratio and the baryon-to-meson ratio $R_{\Lambda_{c}^{+} / D^{0}}$, using for the latter the previous $D^{0}$ measurement [11]. Figure 3 shows the measurement of $R_{\Lambda_{c}^{+} / D^{0}}$ and its comparison with predictions from different nPDF sets, observing hints of a disagreement at high transverse momentum. The results help to constrain the nPDFs, as well as serving as an important input for the hadronisa- 

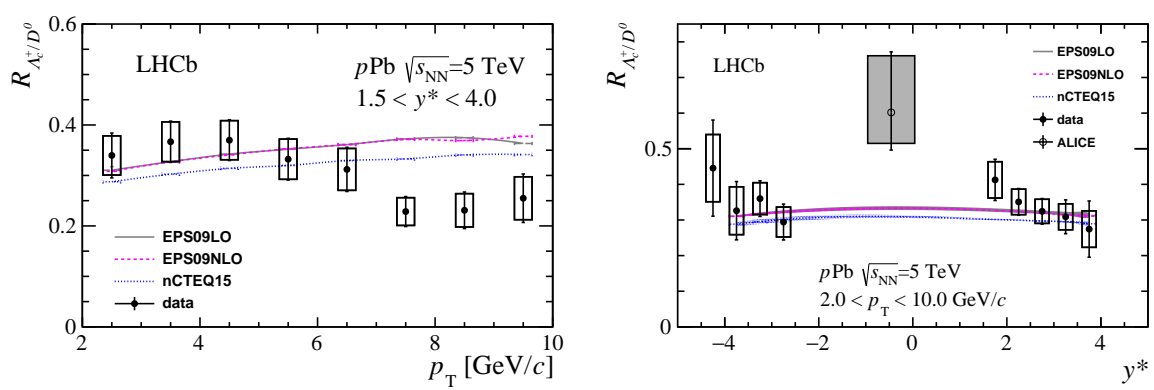

Figure 3: Ratio between $\Lambda_{c}^{+}$and $D^{0}$ production in p-Pb collisions at $\sqrt{s_{\mathrm{NN}}}=5.02 \mathrm{TeV}$, with respect to $y^{*}$ (left) and $p_{\mathrm{T}}$ for the forward configuration (right) [10].
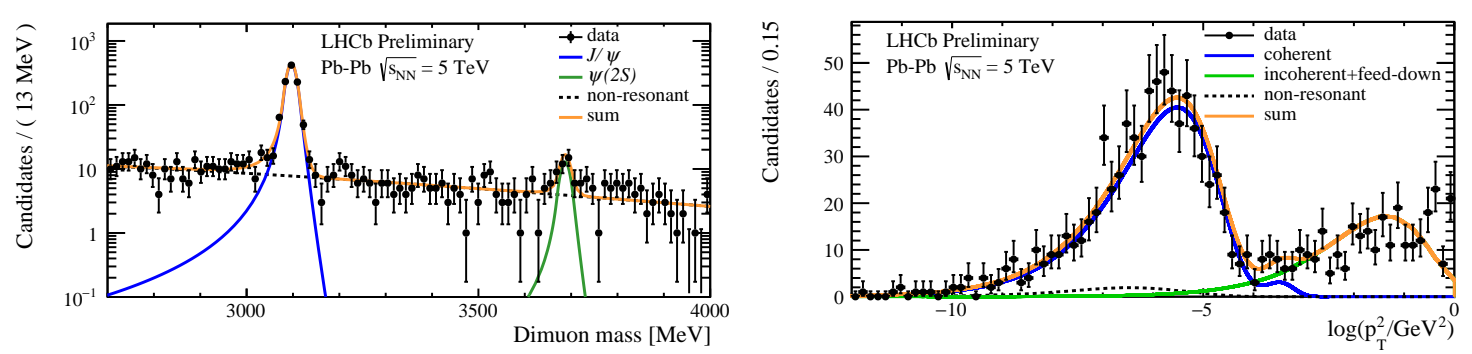

Figure 4: Signal extraction of the coherent $J / \psi$ production in ultra-peripheral $\mathrm{Pb}-\mathrm{Pb}$ collisions at $\sqrt{s_{\mathrm{NN}}}=$ $5 \mathrm{TeV}$, reconstructed with $J / \psi \rightarrow \mu^{+} \mu^{-}$[12].

tion phenomenology.

The scope of LHCb also includes $\mathrm{Pb}$ - $\mathrm{Pb}$ collisions, measuring for its first time the coherent $J / \psi$ production in ultra-peripheral collisions (UPC) at $\sqrt{s_{\mathrm{NN}}}=5.02 \mathrm{TeV}$ using data acquired in 2015, a dataset corresponding to $10 \mu \mathrm{b}^{-1}$ integrated luminosity. Production of vector mesons occurs via photon-gluon interactions, enhanced in $\mathrm{Pb}-\mathrm{Pb}$ due to the high electric charge of the nuclei. The process is particularly interesting to study gluon density down to $x \sim 10^{-5}$ at LHCb. Figure 4 shows the extraction of the $J / \psi$ signal (left figure) and the separation of the coherent and the incoherent contributions (left figure), using templates from the STARlight generator [13]. The main limitation of the study is the limited size of the data sample, which will be overcome with the analysis of the $2018 \mathrm{~Pb}-\mathrm{Pb}$ data sample that accounts for $210 \mu \mathrm{b}^{-1}$ integrated luminosity.

\section{Fixed-target configuration}

A variety of samples has been so far acquired in the the fixed-target configuration, using different gas species (He, Ne and Ar) colliding with proton beams. Depending on the beam energy, the range $\sqrt{s_{\mathrm{NN}}}=68.8-110 \mathrm{GeV}$ is covered. In this configuration, the acceptance in the centreof-mass system is shifted to $-2.8<y^{*}<0.2$, granting access to the high Bjorken- $x$ region in the target nucleon. 

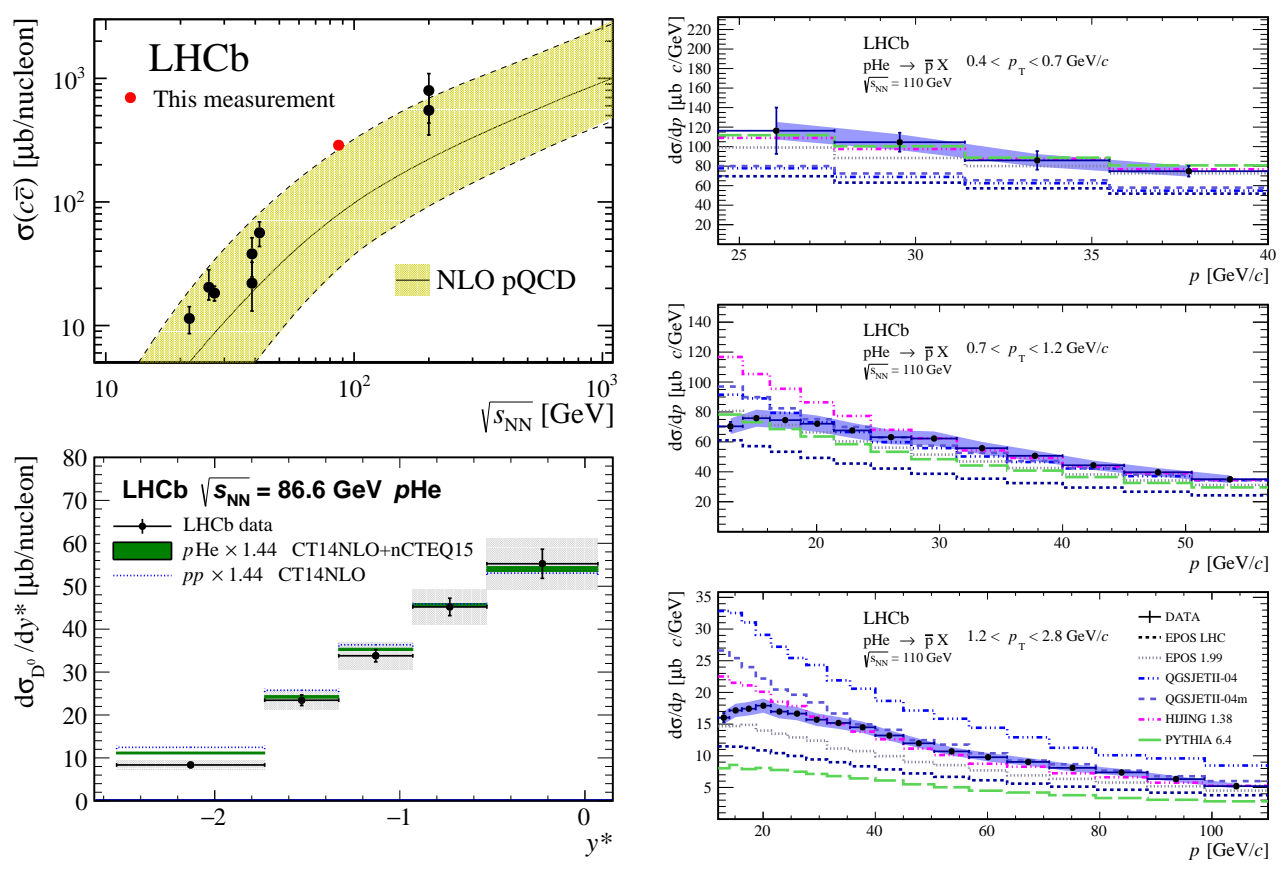

Figure 5: Top left plot: the measurement of the $c \bar{c}$ cross-section from $p$-He collisions at $\sqrt{s_{\mathrm{NN}}}=69 \mathrm{GeV}$ is shown [14]. The result is compared with a NRQCD calculation [15] and other experimental measurements at different energy. Bottom left plot: the differential production cross-section of $D^{0}$ mesons from $p$-He collisions at $\sqrt{s_{\mathrm{NN}}}=86.6 \mathrm{GeV}$ with respect to rapidity is shown [14]. Right plots: the measurement of the $\bar{p}$ production at different transverse momentum bins is shown [16]. The measured value (black dots) is compared with the prediction from several generators, as noted in the figure.

This feature has been exploited in the measurement of charm production in $p$-He and $p$ $\operatorname{Ar}[14]$, as a way to test the intrinsic charm content in the nucleus. Part of the results are shown in figure 5 (left). The measurement does not favour a large contribution of intrinsic charm.

In addition, the antiproton production cross-section in $p$-He collisions at $\sqrt{s_{\mathrm{NN}}}=110 \mathrm{GeV}$ has been determined [16]. The goal of the measurement is to reduce the uncertainty in the measurement of antiprotons in cosmic rays to search for exotic contributions, such as dark matter annihilation. The results are shown in figure 5 (right), where they are compared to different generators. The measurement is significantly more precise than the spread of the models.

\section{Conclusions}

The variety of results obtained so far in $\mathrm{Pb}$ collisions by $\mathrm{LHCb}$ proves the capability to make a remarkable contribution to heavy-ion physics. With the current dataset, many new studies are foreseen. The program will be extended to future LHC runs, taking benefits of the upgraded $\mathrm{LHCb}$ detector [17] and an expected increased luminosity in future heavy-ion runs.

With regard to the fixed-target program, two new samples ( $p$ - $\mathrm{Ne}$ and $\mathrm{Pb}-\mathrm{Ne}$ ) will soon produce new results. For the future runs, a new target device will be installed [18], which will increase the recorded luminosity and will give the possibility to inject other gases such as $\mathrm{H}_{2}$. 


\section{References}

[1] LHCB collaboration, A. A. Alves, Jr. et al., The LHCb Detector at the LHC, JINST 3 (2008) S08005.

[2] LHCB collaboration, R. Aaij et al., LHCb Detector Performance, Int. J. Mod. Phys. A30 (2015) $1530022[1412.6352]$.

[3] LHCB collaboration, R. Aaij et al., Precision luminosity measurements at LHCb, JINST 9 (2014) P12005 [1410.0149].

[4] F. Arleo and S. Peigne, Heavy-quarkonium suppression in p-A collisions from parton energy loss in cold QCD matter, JHEP 03 (2013) 122 [1212. 0434 ].

[5] K. J. Eskola, P. Paakkinen, H. Paukkunen and C. A. Salgado, EPPS16: Nuclear parton distributions with LHC data, Eur. Phys. J. C77 (2017) 163 [1612 . 05741].

[6] E. G. Ferreiro and J.-P. Lansberg, Is bottomonium suppression in proton-nucleus and nucleus-nucleus collisions at LHC energies due to the same effects?, JHEP 10 (2018) 094 [1804.04474].

[7] LHCB collaboration, R. Aaij et al., Measurement of $B^{+}, B^{0}$ and $\Lambda_{b}^{0}$ production in $p \mathrm{~Pb}$ collisions at $\sqrt{s_{\mathrm{NN}}}=8.16 \mathrm{TeV}$, Phys. Rev. D99 (2019) 052011 [1902.05599].

[8] LHCB collaboration, R. Aaij et al., Prompt and nonprompt J/ $\psi$ production and nuclear modification in pPb collisions at $\sqrt{s_{\mathrm{NN}}}=8.16$ TeV, Phys. Lett. B774 (2017) 159 [1706. 07122].

[9] LHCB collaboration, R. Aaij et al., Study of $\Upsilon$ production in pPb collisions at $\sqrt{s_{N N}}=8.16 \mathrm{TeV}$, JHEP 11 (2018) 194 [1810.07655].

[10] LHCB collaboration, R. Aaij et al., Prompt $\Lambda_{c}^{+}$production in $p \mathrm{~Pb}$ collisions at $\sqrt{s_{N N}}=5.02 \mathrm{TeV}$, JHEP 02 (2019) 102 [1809.01404].

[11] LHCB collaboration, R. Aaij et al., Study of prompt $D^{0}$ meson production in $p P b$ collisions at $\sqrt{s_{\mathrm{NN}}}=5 \mathrm{TeV}$, JHEP 10 (2017) 090 [1707.02750].

[12] LHCB collaboration, Study of coherent $J / \psi$ production in lead-lead collisions at $\sqrt{s_{\mathrm{NN}}}=5 \mathrm{TeV}$ with the LHCb experiment, Tech. Rep. LHCb-CONF-2018-003, CERN, Geneva, May, 2018.

[13] S. R. Klein, J. Nystrand, J. Seger, Y. Gorbunov and J. Butterworth, STARlight: A Monte Carlo simulation program for ultra-peripheral collisions of relativistic ions, Comput. Phys. Commun. 212 (2017) 258 [1607.03838].

[14] LHCB collaboration, R. Aaij et al., First Measurement of Charm Production in its Fixed-Target Configuration at the LHC, Phys. Rev. Lett. 122 (2019) 132002 [1810 . 07907].

[15] F. Maltoni et al., Analysis of charmonium production at fixed-target experiments in the NRQCD approach, Phys. Lett. B638 (2006) 202 [hep-ph/ 0601203$].$

[16] LHCB collaboration, R. Aaij et al., Measurement of antiproton production in $\mathrm{pHe}$ collisions at $\sqrt{s_{N N}}=110 \mathrm{GeV}, 1808.06127$.

[17] LHCB collaboration, Framework TDR for the LHCb Upgrade, Tech. Rep. CERN-LHCC-2012-007. LHCb-TDR-12, CERN, Apr, 2012.

[18] LHCB collaboration, LHCb SMOG Upgrade, Tech. Rep. CERN-LHCC-2019-005. LHCB-TDR-020, CERN, May, 2019. 